\title{
A tale of two mechanisms: strain-softening versus strain-hardening in single crystals under small stressed volumes *
}

\author{
H. Bei, ${ }^{1, * *}$ Y.Z. Xia, ${ }^{2}$ R.I. Barabash, ${ }^{1}$ Y.F. Gao ${ }^{1,2}$, \\ ${ }^{1}$ Materials Science and Technology Division, Oak Ridge National Laboratory, Oak Ridge, TN 37831 \\ ${ }^{2}$ Department of Materials Science and Engineering, University of Tennessee, Knoxville, TN 37996
}

\begin{abstract}
Pre-straining defect-free single crystals will introduce heterogeneous dislocation nucleation sources that reduce the measured strength from the theoretical value, while prestraining bulk samples will lead to strain hardening. Their competition is investigated by nanoindentation pop-in tests on variously pre-strained Mo single crystals with several indenter radii ( micrometer). Pre-straining primarily shifts deformation mechanism from homogeneous dislocation nucleation to a stochastic behavior, while strain hardening plays a secondary role, as summarized in a master plot of pop-in strength versus normalized indenter radius.
\end{abstract}

Keywords: nanoindentation pop-in; weakest link; strain hardening

\footnotetext{
* This manuscript has been authored by UT-Battelle, LLC under Contract No. DE-AC05-00OR22725 with the U.S. Department of Energy. The United States Government retains and the publisher, by accepting the article for publication, acknowledges that the United States Government retains a non-exclusive, paid-up, irrevocable, world-wide license to publish or reproduce the published form of this manuscript, or allow others to do so, for United States Government purposes. The Department of Energy will provide public access to these results of federally sponsored research in accordance with the DOE Public Access Plan (http://energy.gov/downloads/doepublic-access-plan).

** Correspondence should be addressed to Hongbin Bei (릉o ornl.gov) or Yanfei Gao (gaoy@ornl.gov)
}

(C) 2015. This manuscript version is made available under the Elsevier user license 
Engineering materials in most structural applications exhibit strengths that are one or several orders of magnitude lower than the theoretical strength. As a large fraction of the Young's or shear modulus, the theoretical strength can only be achieved or approached when the material is pristinely clean and free of any defects, such as in uniaxial tests of carefully grown, micrometer-sized metallic whiskers or fibers [1-3]. For ductile metallic materials, sample preparation and prior thermomechanical treatment usually introduce at least a moderate density of dislocations, and the material strength is governed by the evolution of the dislocation microstructure through a variety of nucleation, propagation, and multiplication processes [4]. A multitude of small scale mechanical experiments have been conducted in recent years to understand the size-dependence of the material strength, which will ultimately bridge the above theoretical strength limit and the bulk flow limit [5-16]. Experimental efforts using micro-pillars machined by focused ion beam (FIB) are mainly focused on searching the scaling relationship between the yield strength and the pillar diameter [5-7]. These studies suffer two major drawbacks, one being the potential sample surface damage due to FIB treatment [6] and the other being the large variation of data that prevent a meaningful simple scaling [7-9]. An alternative testing method that avoids the cumbersome FIB process or the whisker/fiber growth is the use of instrumented nanoindentation at sub-micron scales [11-16]. For carefully electro-polished surfaces (thus removing surface oxides and other contaminating surface layers), the loaddisplacement curves usually exhibit sudden displacement bursts (or called pop-ins), and the popin strength is an indication of the material yield strength in the corresponding small stressed volumes.

Similar to the extensively studied uniaxial tests, the pop-in strength has two asymptotes as shown in Fig. 1(a). When the stressed volume is free of any pre-existing defects, the pop-in 
corresponds to the homogeneous nucleation of dislocation at the theoretical strength. At the bulk limit, the pop-ins are difficult to observe and the deviation from the elastic load-displacement curves is governed by the bulk yield stress of the material. In the intermediate stage between these two asymptotes, the pop-in strength shows a significant scattering. Primarily because of the random nature of the distribution of pre-existing defects, chances exist that the stressed volume may contain no or a small number of pre-existing defects, so that the pop-in strength can vary from theoretical strength to the bulk stress. In principle, it should be noted that any type of mechanical tests in the intermediate scale regime should find similar scattering, which is however rarely studied systematically in micro-pillar tests because the tedious sample preparation by FIB prevents such statistical measurements. In contrast, nanoindentation pop-in tests $[3,12,13,15]$ can be conveniently done in a small surface area and thus be utilized to study the transition of governing mechanisms with respect to the change of stressed volume size, defect density, and other parameters.

One question that arises naturally from observing the deformation characteristics in Fig. 1(a) is what the effects of pre-strains are on the material strength. At the bulk limit, pre-strain will lead to the increase of dislocation density, and correspondingly the yield stress will increase - this is the strain hardening mechanism when the dislocation density increases [4]. Near the theoretical stress limit, however, the pre-existing defects introduced by pre-straining before the pop-in tests will reduce the pop-in stress. These defects will act as the weakest links that change the homogeneous dislocation nucleation (that requires the theoretical stress) to a heterogeneous dislocation nucleation mechanism (such as Frank-Read source that requires a low stress). This is a strain softening mechanism when dislocation density increases. Although the above two mechanisms operate at different limits, they both contribute in the intermediate scale regime and 
in principle one can tune their relative significance by tuning the degree of pre-strain. Consequently, in this paper, a synergy amongst nanoindentation pop-in tests, synchrotron x-ray measurements (for the characterization of dislocation density), and theoretical modeling will be employed to identify the dominant regimes of these two mechanisms and to study whether and how they can be separated.

Molybdenum single crystals used in this study (from Alfa Aesar) had been electron beam floating zone refined and had a purity of $99.99 \%$. The typical interstitials in electron beam refined Mo are carbon ( 0.5 ppm) and oxygen (5 ppm) [17]. All specimens for nanoindentation tests were cut from the same Mo single crystal rod ( $\sim 11 \mathrm{~mm}$ in diameter and $100 \mathrm{~mm}$ in length) by using electron-discharge-machining. Before cutting, the Mo single crystal rod was homogenized at $1600{ }^{\circ} \mathrm{C}$ for 4 hours in a vacuum furnace. Nanoindentation specimens were diskshaped, of $2 \mathrm{~mm}$ thickness, and with surface normal in $<100>$ directions. Three disks were compressed in room temperature with pre-strains (ratios of the reduction of thickness to the initial thickness) of $1.5 \%, 5 \%$, and $13 \%$. Together with no pre-strained disk $(0 \%)$, these specimens were mounted in epoxy, ground and polished with standard metallographic procedure. The final polishing step was conducted electrochemically at $\sim 10 \mathrm{~V}$ in a 12.5 vol. $\% \mathrm{H}_{2} \mathrm{SO}_{4}$ methanol solution. In order to cover the whole range of deformation behavior in Fig. 1(a), nanoindentation tests were performed in a Nanoindenter XP (MTS Nano Instruments, Oak Ridge, TN) by using a number of indenters, including two Berkovich diamond indenters with effective tip radii $R$ of 115 and $210 \mathrm{~nm}$, five diamond spherical tips with effective radii $R$ of 0.58 , $1.5,3.66,6.9,18 \mu \mathrm{m}$. The tip radii were calibrated by the method in [18], which considers the contribution of the machine stiffness. All tests were performed in the continuous stiffness mode (CSM) with a constant rate of $\dot{P} / P=0.05 \mathrm{~s}^{-1}$. A total number of 36 indents were made with each 
indenter so as to achieve sufficient statistical variations, and these indents were placed far from one another to avoid mechanical interference.

Before presenting our nanoindentation pop-in measurements, we identify the relationship between pre-strain and dislocation density in these samples by the Polychromatic X-ray Microdiffraction (PXM) technique. Data collection with PXM was carried out at the beamline ID-34-E at the Advanced Photon Source (Argonne, IL), using a modified Laue diffraction technique. It allows for true 3D mapping of crystalline phase, orientation, elastic strain and plastic deformation with less that $0.5 \mu \mathrm{m}$ spatial resolution [19-21]. Laue patterns from pre-strained Mo single crystals consist of both streaked and broad Laue spots, with the former governed by the formation of geometrically necessary dislocations (GNDs) and deviatoric strain and with the latter depending on the total dislocation density. Measurements were performed in several locations for appropriate statistical sampling. The broadening of the $\{006\}$ Mo reflection is shown in Fig. 1(b) for annealed, 1.5\% pre-strained, and 5.0\% pre-strained samples, where $Q$ is reciprocal to the spacing of $\{006\}$ lattice planes. The method in [19] was employed to calculate the total dislocation density, as given in Table I. The 13\% pre-strained sample was not utilized because it was found that the dislocation arrangement became inhomogeneous - dislocations organized into specific patterns with different kind of dislocation walls with high dislocation density and some part of randomly distributed dislocations between them.

Two representative load-displacement curves of (100) Mo single crystals are shown in Fig. 2. With the use of an indenter radius of $1.5 \mu \mathrm{m}$ on the annealed sample in Fig. 2(a), a giant pop-in excursion was found at the load of $\approx 24 \mathrm{mN}$ and the displacement of $\approx 140 \mathrm{~nm}$, respectively. The contact response before the pop-in can be found to be purely elastic with the relationship between load $P$ and displacement $h$ following the Hertzian solution, 


$$
P=\frac{4}{3} E_{r} \sqrt{R} h^{3 / 2},
$$

where the reduced modulus $E_{r}$ can be calculated from the elastic constants of the indenter and the specimen [22]. Using $R=1.5 \mu \mathrm{m}$ and $E_{r}=270 \mathrm{GPa}$, [13] we obtain the theoretical curve, $P=0.0139 \times h^{3 / 2}$, which is plotted as a solid curve (Hertzian) in Fig. 2(a). The good agreement between the experimental load-displacement curve up to pop-in and Hertzian analysis confirms that before the pop-in, the deformation underneath the indenter is fully elastic. To compare all the samples under various indenter radii, we convert the pop-in load to the critical shear stress that represents the shear strength of the material. Following Ref. [3], we find the following

$$
\tau_{\max }=0.31 \times\left(\frac{6 P_{p o p-i n} E_{r}^{2}}{\pi^{3} R^{2}}\right)^{1 / 3}
$$

For the indenter size of $1.5 \mu \mathrm{m}$, all 36 indentations on the annealed sample show pop-ins, and the maximum shear stress at pop-in calculated by using Eq. (2) is found to vary from 9.0 to 16.1 $\mathrm{GPa}$. The upper value $(16.1 \mathrm{GPa})$ for this indenter is $\sim 1 / 8$ its shear modulus, which agree very well with the theoretical strength obtained by ab initio calculations using density functional theory [3]. We also point out that uniaxial tests of fibers or wires will have a reduced upper value of shear strength because of dislocation nucleation from surfaces or ledges [3].

In Fig. 2(b), a representative load-displacement curve is given for $1.5 \%$ pre-strained sample using the same indenter with radius $R=1.5 \mu \mathrm{m}$. The $\mathrm{P}-\mathrm{h}$ curve before pop-in is still elastic, indicating that the pop-in still corresponds to a plasticity initiation mechanism. Now the pop-in load is significantly lower (by a factor of about 25), or equivalently, the maximum shear stress is about three times lower from Eq. (2). 
Results in Fig. 2 and the schematic illustration in Fig. 1(a) suggest that the increase of indenter radius and the pre-strain both reduce the pop-in strength. Thus Fig. 3(a) presents our systematic tests of a number of indenter radii and two pre-strain levels. The three types of pop-in mechanisms in Fig. 1(a) can now be described by the cumulative pop-in probability as a function of the pop-in stress. When the stressed volume is small such as $R=115 \mathrm{~nm}, 210 \mathrm{~nm}$, and $580 \mathrm{~nm}$ in Fig. 3(b), the pop-in stress data have small variation, and the cumulative pop-in probability curve is steep. In this case, the statistical variation is due to thermal activation of homogeneous dislocation nucleation, and its survivability is given by

$$
q_{\text {homo }}=\exp \left(-\dot{n}_{0} \int_{0}^{P_{\text {pop-in }}} \frac{d P}{\dot{P}} \int_{\Omega} \exp \left\{-\frac{\Delta \Pi\left(\tau_{\text {shear }}\right)}{k_{B} T}\right\} d \Omega\right),
$$

where survivability $q$ relates to cumulative probability $f$ by $f=1-q$. The derivation is based on the transition state theory $[3,11]$, in which the dislocation nucleation rate is given by $\dot{n}_{0} \exp \left(-\Delta \Pi / k_{B} T\right)$. In the above, $\dot{n}_{0}$ is the characteristic nucleation rate, $k_{B}$ is Boltzmann constant, $T$ is absolute temperature, and $d \Omega$ is the differential volume. The corresponding activation energy $\Delta \Pi\left(\tau_{\text {shear }}\right)$ depends on the shear stress $\tau_{\text {shear }}$, as given in our previous study $[12,15]$.

With the increase of stressed volume, the scatter in pop-in stress increases dramatically as shown by the no pre-strained sample at $R=1.5 \mu \mathrm{m}$ and $6.9 \mu \mathrm{m}$, the $1.5 \%$ pre-strained sample at $R=115 \mathrm{~nm}$ and $210 \mathrm{~nm}$, and the $5 \%$ pre-strained sample at $R=115 \mathrm{~nm}$. For these cases, the cumulative pop-in probability curves show significant tails at low stress, which arises from a heterogeneous dislocation nucleation mechanism. Following $[8,13,15,16]$, we assume that the distribution of the pre-existing defects obeys a Poisson's function, so that the pop-in due to 
heterogeneous mechanism will occur if the stressed volume contains such a pre-existing defect. That is,

$$
q_{\text {hetero }}=\exp \left(-\rho_{\text {defect }} V_{d}\right)
$$

where $\rho_{\text {defect }}$ is the density of pre-existing defects or heterogeneous dislocation nucleation sites (given in the unit of $1 /$ volume), and $V_{d}$ is the stressed volume size in which the shear stress higher than a activation strength, $\tau_{\text {defect }}$. For instance, this could be related strength of the FrankRead source. These two parameters, $\rho_{\text {defect }}$ and $\tau_{\text {defect }}$, are to be fitted to experimental curves. The convolution of the above two mechanisms in Eqs. (3) and (4) gives a total cumulative probability, $f_{\text {total }}=1-q_{\text {hetero }} \times q_{\text {homo }}$, which is represented by the solid curves in Fig. 3 .

The transition from homogeneous nucleation, to intermediate stochastic behavior, and to bulk behavior can be observed from the steep curves near $R=115 \mathrm{~nm}$, the large-tailed curves such as $R=1.5 \mu \mathrm{m}$, and the steep curves again near $R=18 \mu \mathrm{m}$ in Fig 3 (b) for the annealed single crystal. For the $1.5 \%$ pre-strained case in Fig. 3(c), the smallest indenter at $R=115 \mathrm{~nm}$ already exhibits the intermediate stochastic behavior, because a higher $\rho_{\text {defect }}$ (about $10 \mu \mathrm{m}^{-3}$ which is about four orders of magnitude higher than that of the no pre-strained sample) will easily lead to sampling a pre-existing defect as described by Eq. (4). In other words, this is the strain softening mechanism due to the introduction of the weakest links upon pre-straining. This phenomenon is even more obvious in Fig. 3(d) for the 5\% pre-strain sample by comparing the cumulative pop-in probability curve at $R=115 \mathrm{~nm}$ with its counterparts in Figs. 3(b) and 3(c). A caution should be paid to the difference between $\rho_{\text {defect }}$ in Eq. (4) and the dislocation density in Table I. The defect density represents the density of the heterogeneous dislocation nucleation sources, such as the Frank-Read type, so that it should be lower than the total dislocation density. In other words, the 
pre-existing defect spacing (being about 5.7, 0.38 , and $0.26 \mu \mathrm{m}$ for no pre-strained, $1.5 \%$ prestrained and 5\% pre-strained samples from Eq. (4) should be larger than the dislocation spacing (being about 3.09, 0.043, $0.025 \mu \mathrm{m}$, respectively, from PXM measurement) in Table I.

In principle, all these three sets of data in Fig. 3 should converge to their corresponding bulk limit, as nicely described by the fitted $\tau_{\text {defect }}$ in Table I. This parameter should be proportional to the hardness value in Table I, therefore leading to about $20 \%$ increase of the bulk limit, or equivalently from $0.644 \mathrm{GPa}$ to $0.773 \mathrm{GPa}$. However, such an increase will not be noticed on the vast scale from $0.5 \mathrm{GPa}$ to $20 \mathrm{GPa}$ in Fig. 3(a), as shown in the inset in Fig. 4. It should be pointed out that the dependence of indentation hardness on indentation depth and prestrain $[23,24]$, i.e., indentation size effects, is commonly studied at indentation depths much larger than our tests.

In summary, our findings in this paper can be represented in the master plot of pop-in stress versus $R \rho_{\text {defect }}^{1 / 3}$ in Fig. 4, where the range of the data scatter is marked by the $10 \%$ and $90 \%$ probability curves. The increase of pre-strain is equivalent to increase the stressed volume size, both of which will merely increase the probability of finding the weakest links that trigger the heterogeneous pop-in mechanism. This strain softening mechanism dominate over a wide range of sample size and defect density, whereas the strain hardening mechanism that is often observed in bulk sizes can only be observed in a small window (see the inset of Fig. 4). These findings suggest that one be cautious with the interpretation of the size-dependence of material strength; the stochastic behavior should be characterized before concluding any scaling relationship of material strength with respect to their sample size. 
This research was supported by the U.S. Department of Energy, Office of Science, Basic Energy Sciences, Materials Sciences and Engineering Division. X-ray microbeam measurements were performed at ID-34-E at the Advanced Photon Source, which was supported by the U.S. Department of Energy, Office of Science, Basic Energy Sciences, Scientific Users Facilities Division. 


\section{References}

1. $\quad$ S.S. Brenner, J. Appl. Phys. 27, 1484-1491 (1956).

2. S.S. Brenner, J. Appl. Phys. 28, 1023-1026 (1957).

3. H. Bei, Y.F. Gao, S. Shim, E.P. George, G.M. Pharr, Phys. Rev. B 77, 060103(R) (2008).

4. A. H. Cottrell, Dislocations and Plastic Flow in Crystals. Clarendon Press, Oxford (1953).

5. M.D. Uchic, D.M. Dimiduk, J.N. Florando, W.D. Nix, Science 305, 986 (2004).

6. D. Kiener, C. Motz, M. Rester, M. Jenko, G. Dehm, Mater. Sci. Eng. A 459, 262 (2007).

7. A. Bharathula, K.M. Flores, Acta Mater. 59, 7199 (2011).

8. R. Maaß, P.M. Derlet and J.R. Greer, Small 11, 341 (2015).

9. P.E. Loya, Y.Z. Xia, C. Peng, H. Bei, P. Zhang, J. Zhang, E.P. George, Y.F. Gao, J. Lou, Appl. Phys. Lett. 104, 251909 (2014).

10. X.Y. Zhao, D.J. Strickland, P.M. Derlet, M. He, Y.J. Cheng, J. Pu, K. Hattar, D.S. Gianola, Acta Mater. 88, 121 (2015).

11. C.A. Schuh, A.C. Lund, J. Mater. Res. 19, 2152 (2004).

12. T.L. Li, Y.F. Gao, H. Bei, E.P. George, J. Mech. Phys. Solids 59, 1147 (2011).

13. J.R. Morris, H. Bei, G.M. Pharr, E.P. George, Phys. Rev. Lett. 106, 165502 (2011).

14. I.C. Choi, Y.K. Zhao, B.G. Yoo, Y.J. Kim, J.Y. Suh, U. Ramamurty, J.I. Jang, Scripta Mater. 66, 923 (2012).

15. T.L. Li, H. Bei, J.R. Morris, E.P. George, Y.F. Gao, Mater. Sci. Technol. 28, 1055 (2012).

16. W.D. Li, H. Bei, Y. Tong, W. Dmowski, Y.F. Gao, Appl. Phys. Lett. 103, 171910 (2013).

17. A. Kumar, B.L. Eyre, Proc. R. Soc. Lond. A 370, 431 (1980).

18. W.D. Li, H. Bei, J. Qu, Y.F. Gao, J. Mater. Res. 28, 1903 (2013).

19. R.I. Barabash, H. Bei, Y.F. Gao, G.E. Ice, E.P. George, J. Mater. Res. 25, 199 (2010).

20. R.I. Barabash, H. Bei, Y.F. Gao, G.E. Ice, Acta Mater. 58, 6784 (2010).

21. R.I. Barabash, H. Bei, Y.F. Gao, G.E. Ice, Scripta Mater. 64, 900 (2011).

22. Y.F. Gao, G.M. Pharr, Scripta Mater. 57, 13 (2007).

23. Y.Y. Lim, M.M. Chaudhri, Phil. Mag. A 79, 2979 (1999).

24. G.M. Pharr, E.G. Herbert, Y.F. Gao, Annu. Rev. Mater. Res. 40, 271 (2010). 


\section{Tables}

Table I Nanoindentation modulus and hardness obtained by experiments, dislocation density measured by the polychromatic x-ray micro-diffraction (PXM) technique, and the pre-existing defect density and strength $\left(\rho_{\text {defect }}, \tau_{\text {defect }}\right)$ obtained by the unified model in Eqs (3) \& (4) for annealed and pre-strained Mo (100) single crystals. The theoretical strength is $\tau_{t h}=16.1 \mathrm{GPa}$.

\begin{tabular}{cccc}
\hline Pre-strain & $0 \%$ & $1.5 \%$ & $5.0 \%$ \\
\hline Modulus (GPa) & 327 & 327 & 327 \\
Hardness (GPa) & $2.22( \pm 0.02)$ & $2.54( \pm 0.01)$ & $2.67( \pm 0.02)$ \\
Disl. density $\left(\mathrm{cm}^{-2}\right)(\mathrm{PXM})$ & $1.047 \times 10^{7}$ & $5.349 \times 10^{10}$ & $1.599 \times 10^{11}$ \\
& & & \\
Disl. spacing $(\mu \mathrm{m})(\mathrm{PXM})$ & 3.09 & 0.0432 & 0.0250 \\
$\rho_{\text {defect }}\left(\mu \mathrm{m}^{-3}\right)(\mathrm{Eqs.} 3 \& 4)$ & 0.0053 & 17.5 & 55 \\
$\tau_{\text {defect }}$ & 5.7 & 0.38 & 0.26 \\
& $0.04 \tau_{\text {th }}$ & $0.046 \tau_{\text {th }}$ & $0.048 \tau_{\text {th }}$ \\
\hline
\end{tabular}




\section{Figure Captions}

Figure 1 (a) Schematic diagrams showing different dominant mechanisms for nanoindentation pop-ins, being homogeneous nucleation at small stressed volumes, stochastic behavior at intermediate sizes, and conventional plasticity at large sizes. (b) Synchrotron X-ray results of the peak broadening of $\{006\}$ Mo reflection in the three samples, from which the dislocation densities can be calculated as given in Table I.

Figure 2 Representative load-displacement (P-h) curves, using indenter radius of $R=1.5$ $\mu \mathrm{m}$, show clear pop-in for the annealing sample in (a) and for the $1.5 \%$ prestrained sample in (b). Prior to the pop-ins, the P-h curves are nicely described by the theoretical Hertzian contact behavior.

Figure 3 Critical shear stress at pop-in as a function of the indenter radius for the three specimens in (a), and the corresponding cumulative pop-in probability versus the critical shear stress at pop-in for no pre-strained specimen in (b), $1.5 \%$ prestrained sample in (c), and 5\% pre-strained sample in (d). Legends in (b)-(d) are the indenter radii in $\mu \mathrm{m}$.

Figure 4 A master plot of the critical shear stress at pop-in as a function of the normalized indenter radius (normalized by $\rho_{\text {defect }}$ ) clearly exhibits the transition from thermally activated homogeneous dislocation nucleation (near the theoretical strength) to stochastic behavior and then to the bulk strength. The increase of the pre-strain (thus increasing $\rho_{\text {defect }}$ ) is equivalent to increasing the stressed volume size, leading to large variation of pop-in strength. Strain hardening affects the bulk limit as depicted by the inset. Solid and dashed curves indicate $10 \%$ and $90 \%$ cumulative probabilities. 
(a)

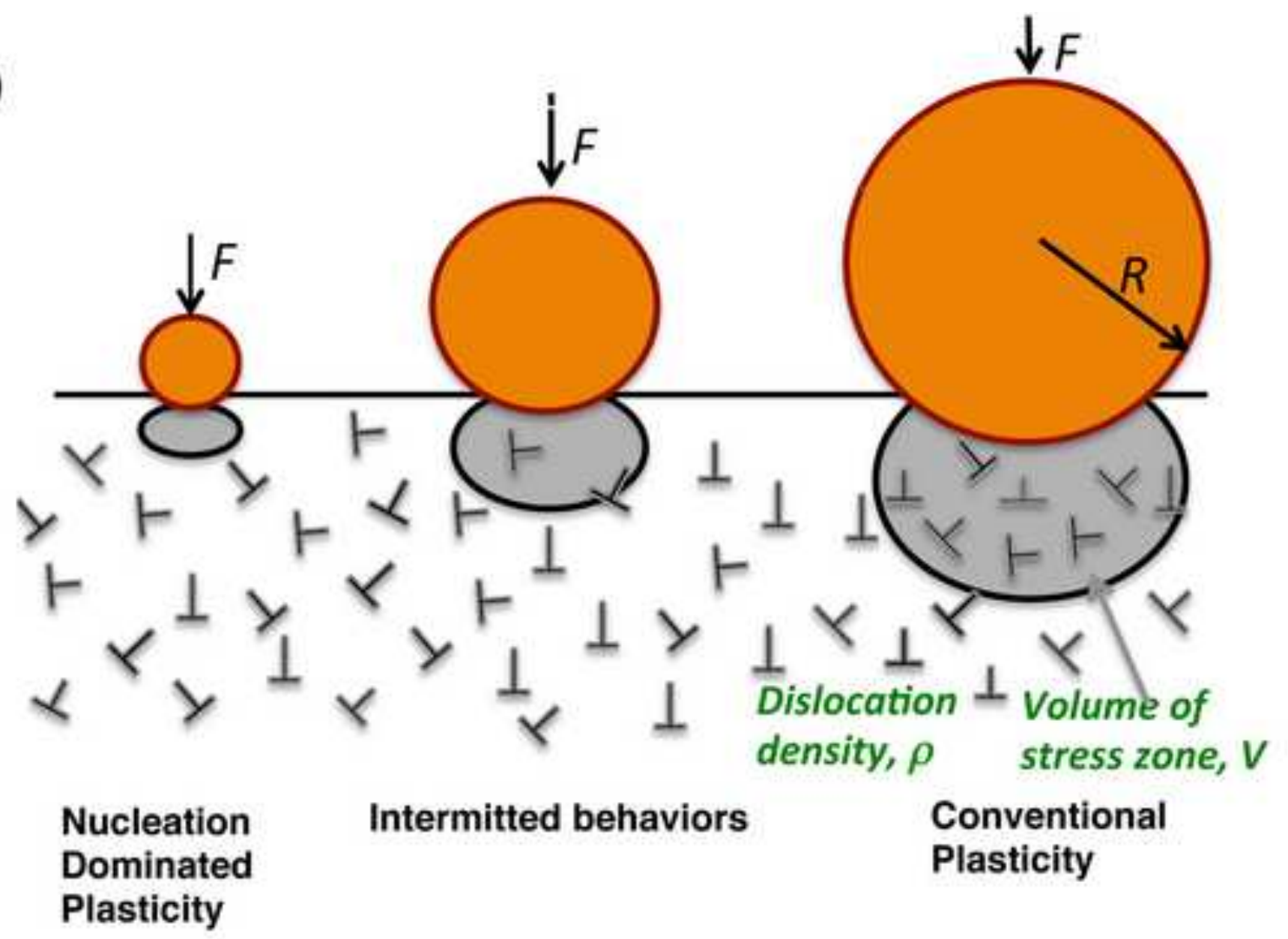

(b)

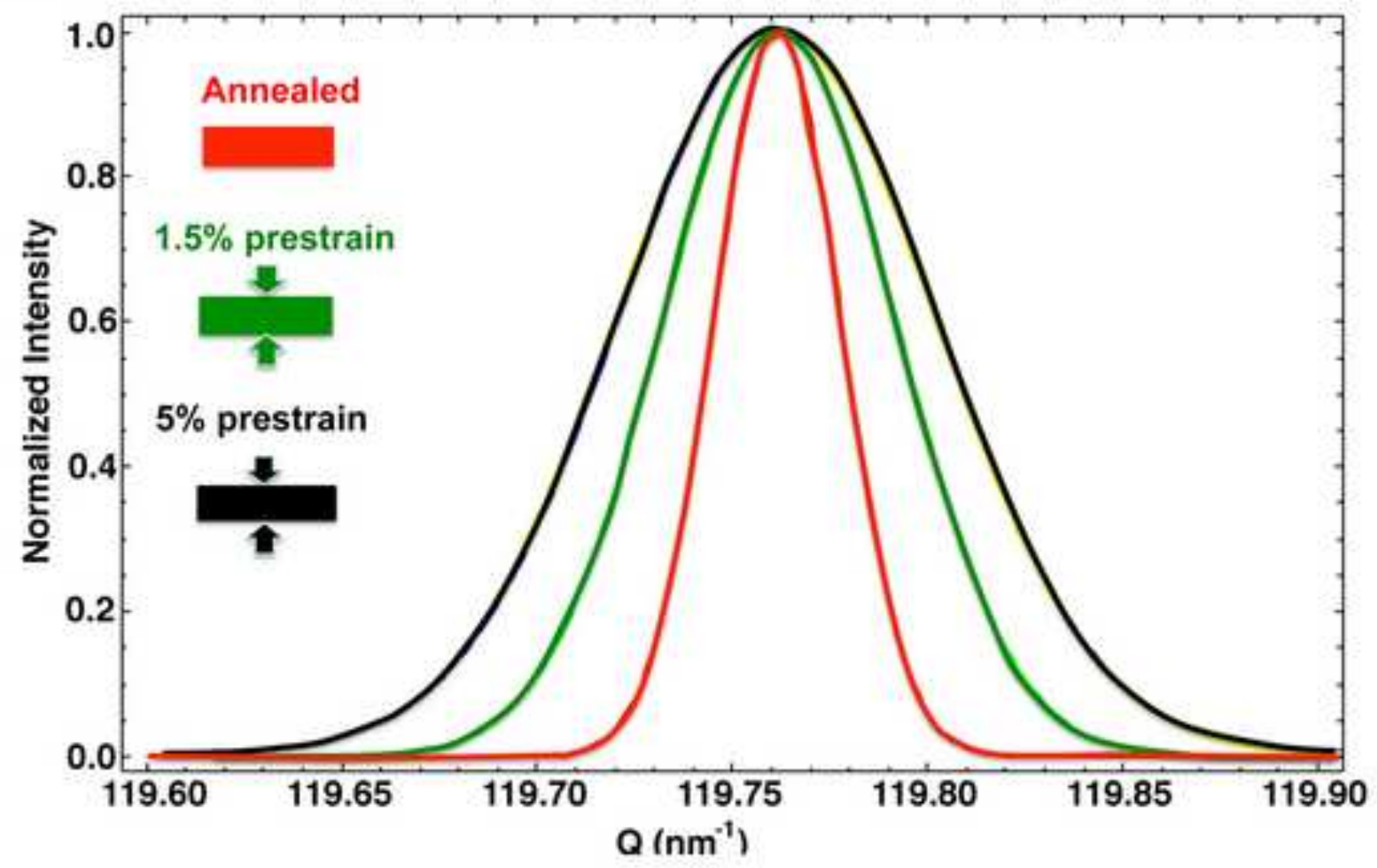



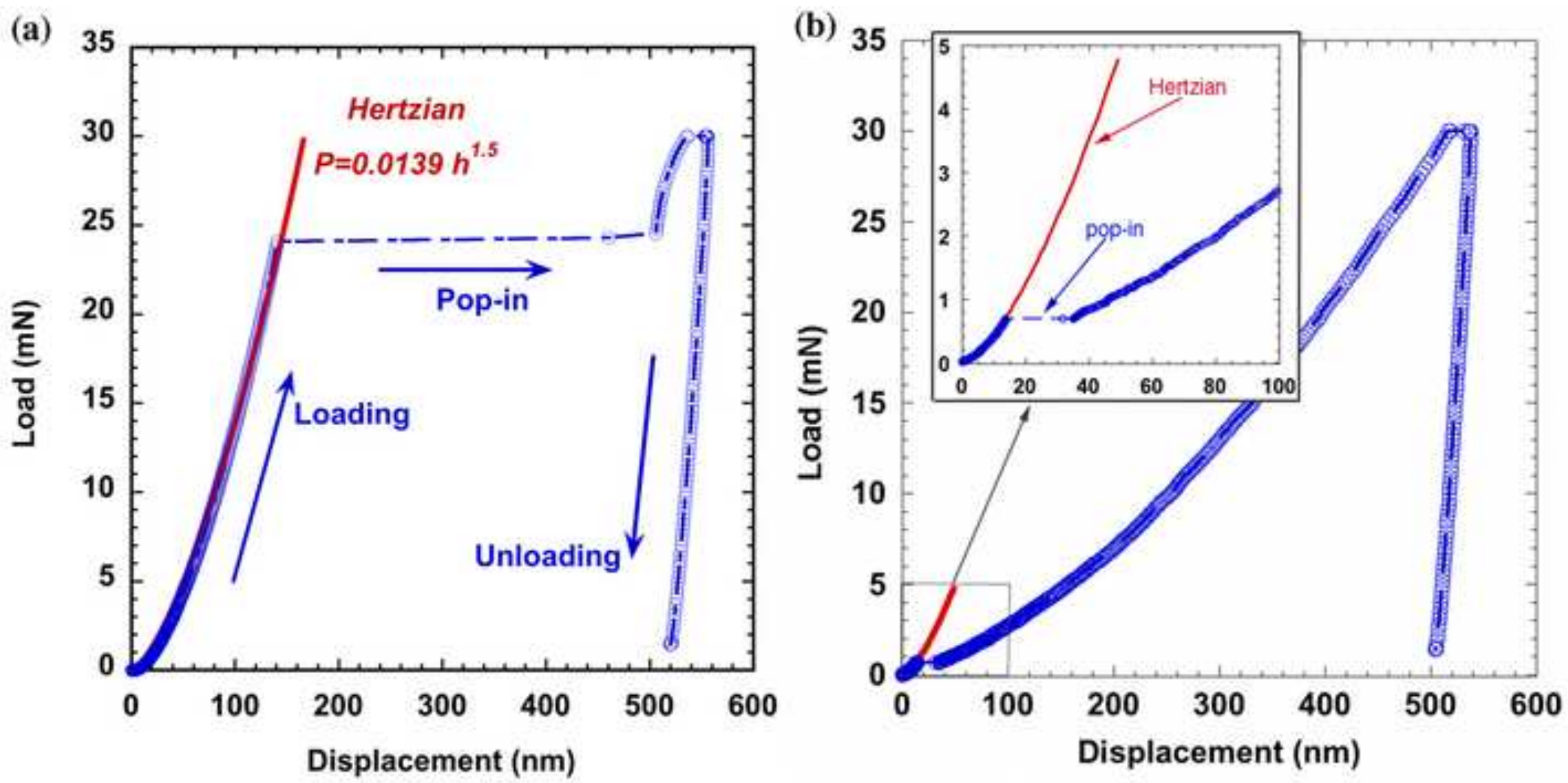

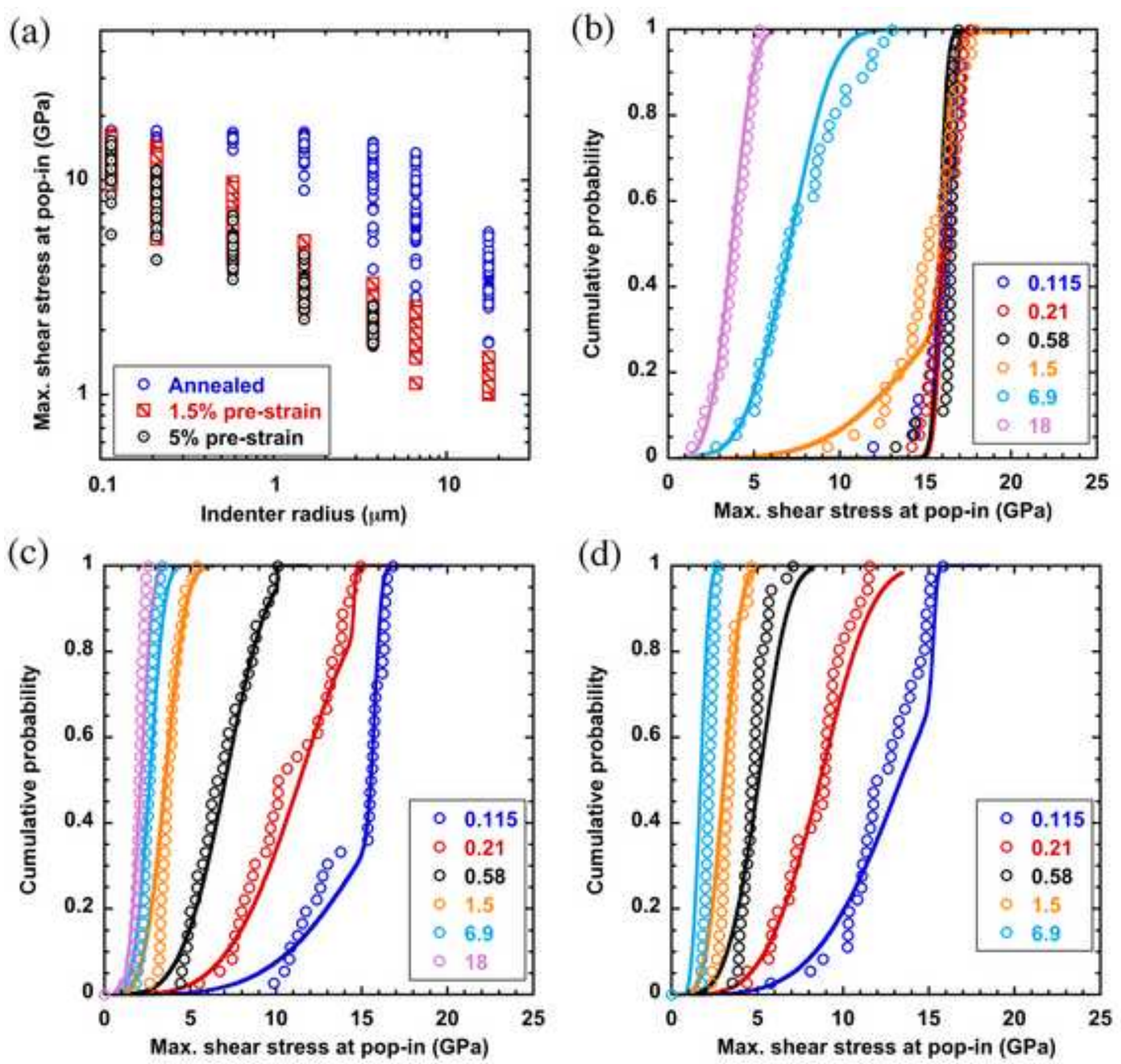


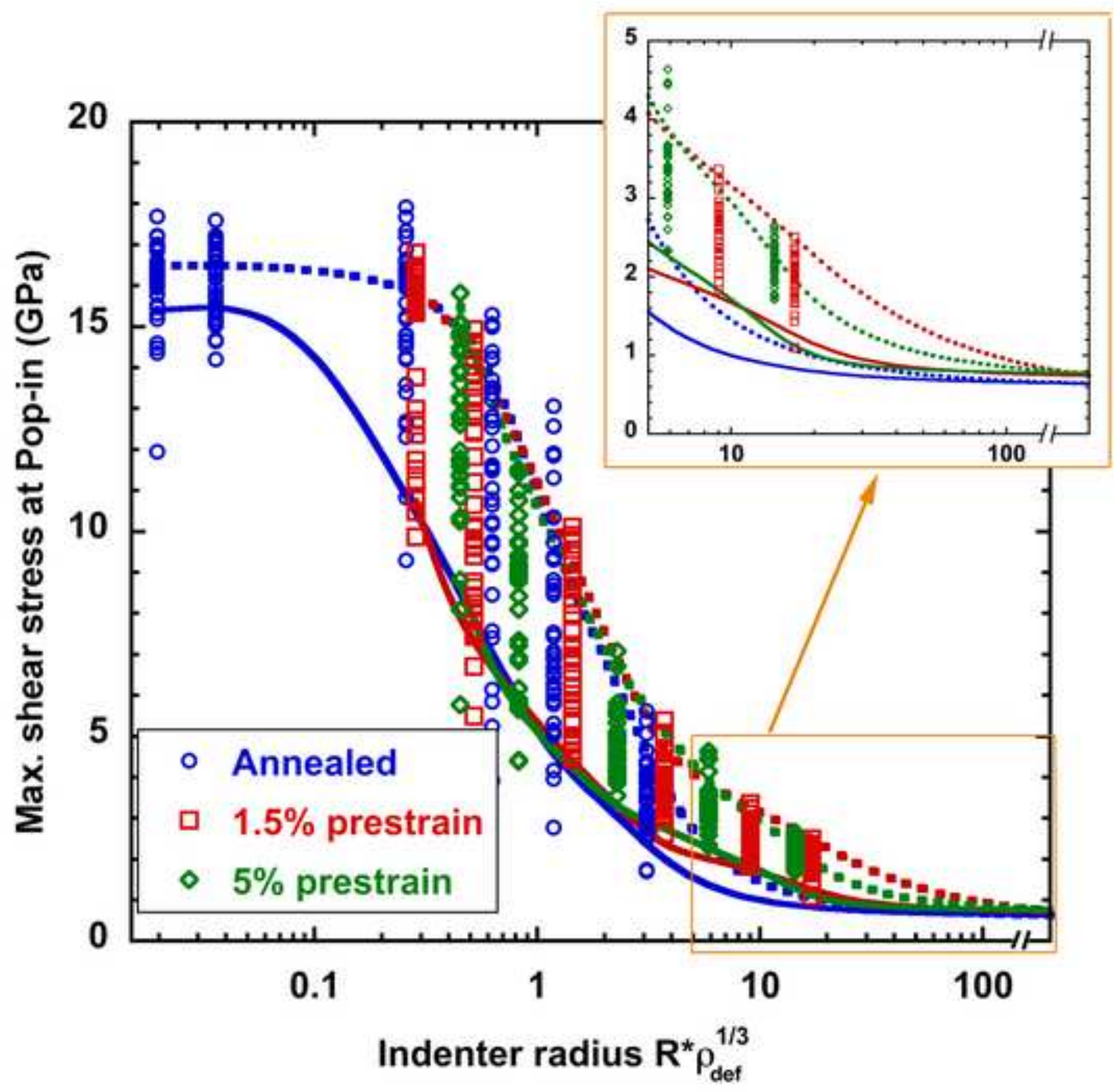


Plasticity Initiation vs. Strain Hardening

\section{Under Small Stressed Volumes}

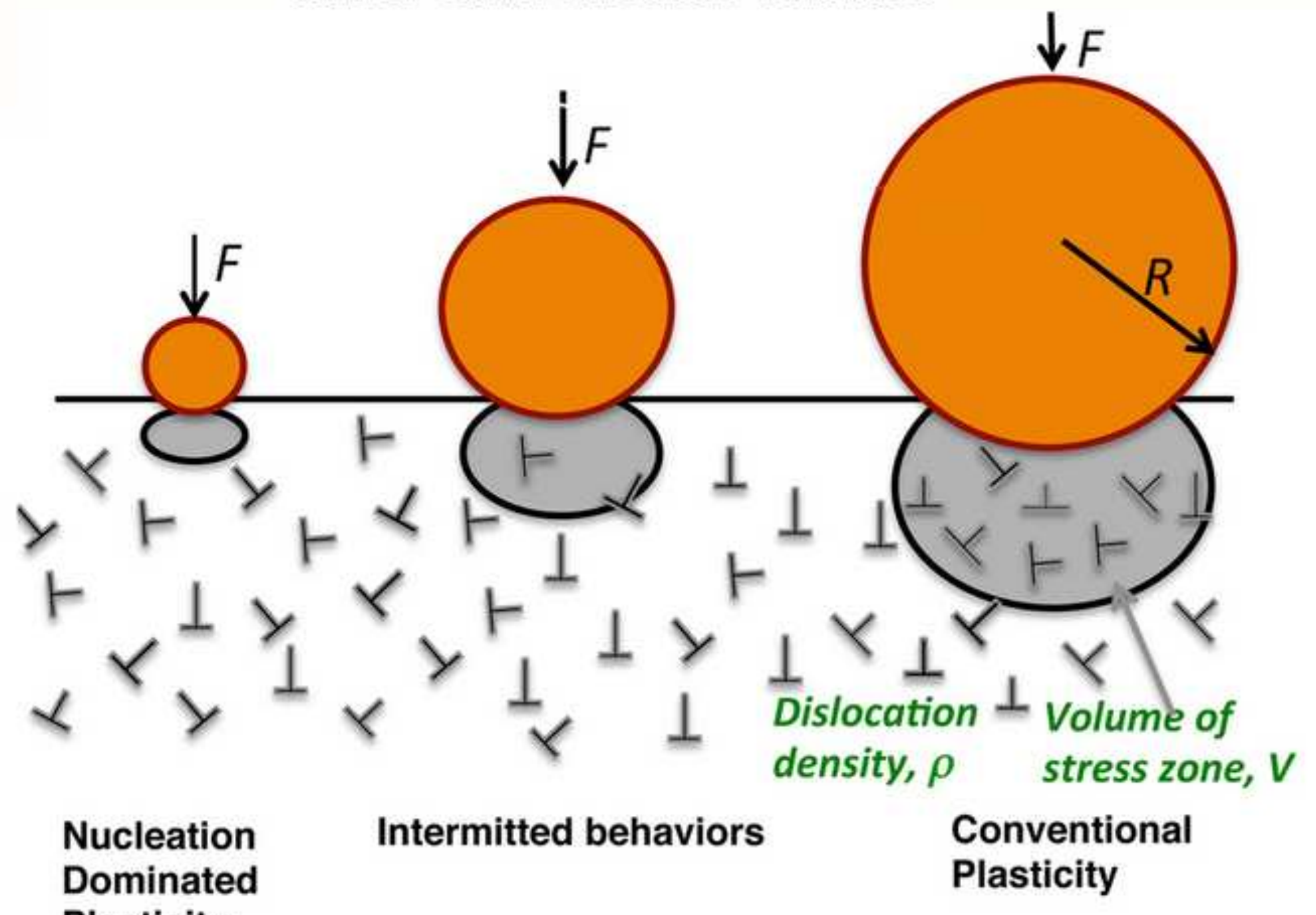

Plasticity 